\title{
ABPr en la enseñanza superior de la didáctica musical
}

\author{
Adrien Faure-Carvallo ${ }^{a}$ y Diego Calderón-Garrido \\ Universidad de Barcelona (aadrienfaure@ub.edu y ${ }^{b}$ dcalderon@ub.edu)
}

\section{\$EWWFW}

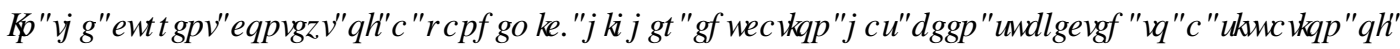

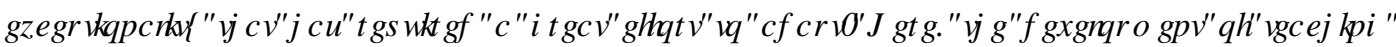

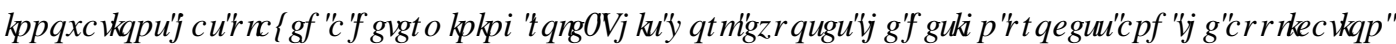

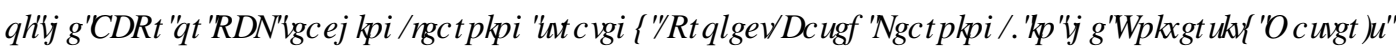

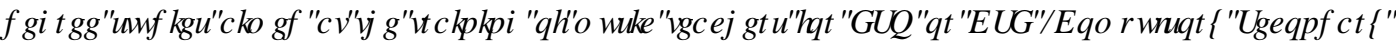

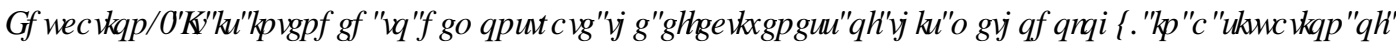

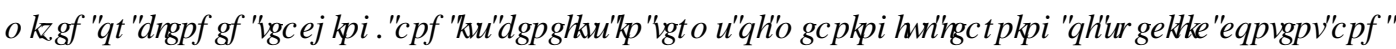
WKHDFTXLMURQRIWWDQYHWOMNCOIQLUJ KHUHCXFDMRQ]

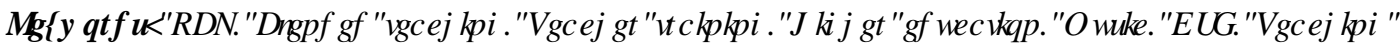
IQQRYDMRQI

]

\section{HXP HQ}

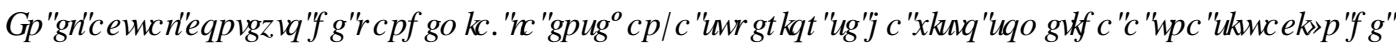

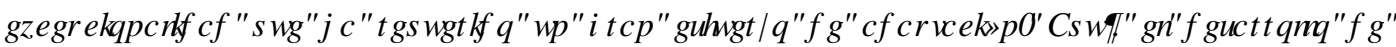

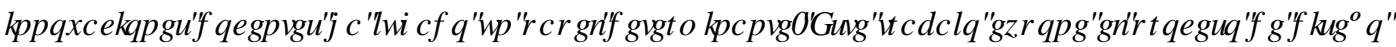

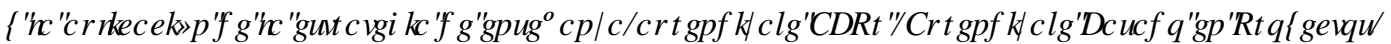

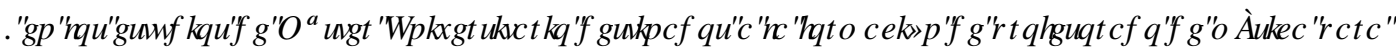

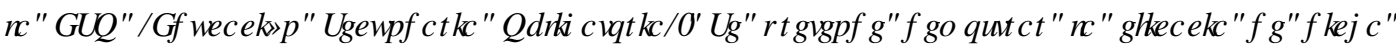

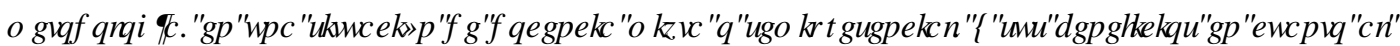

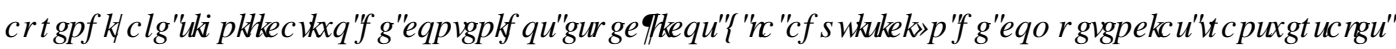
HQ(D)HCXFDFIYQXSHURU

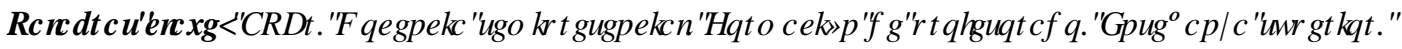
$0 \sim \operatorname{VFDI}(62$ प, QQRYDFIYQQRFHQMUI

○

\section{Introducción}

Los estudios universitarios destinados a la formación de profesorado llevan tiempo promoviendo el uso de metodologías docentes innovadoras (Finkel y Carpintero, 2019). Actualmente, a cualquier estudiante de los Grados o $0 D W H V$ de Educación Infantil, Primaria o Secundaria, se le preparará para afrontar un futuro laboral en el que deberá aplicar nuevas formas de enseñar, adaptadas a los tiempos en los que vivimos, y que suponen sin duda un avance positivo en el devenir de la educación. Paradójicamente, el tradicional formato de exposición magistral sigue siendo el más empleado en las aulas de las universidades de nuestro país, junto con las prácticas a través de las TIC's (Losada y García, 2018). Además, la situación de pandemia, que ha sacudido al mundo durante los últimos meses, ha puesto entre la espada y la pared al HMDWVTXR educativo y ha evidenciado la necesidad urgente de renovar las metodologías pedagógicas en la enseñanza superior. 
En este trabajo se presenta una experiencia de docencia mixta, que ha combinado clases presenciales, clases en línea y sesiones de aprendizaje autónomo, y en la cual se ha aplicado la metodología ABPr -Aprendizaje Basado en Proyectos-. La innovación ha sido aplicada en la asignatura Didáctica de la Expresión y la Creación Musicales (código: 566 002; 2,5 créditos), del Master de Formación del Profesorado de Secundaria Obligatoria y Bachillerato de la Universidad de Barcelona. Se trata de una asignatura optativa, que se ha desarrollado en modalidad mixta -las clases, parte de la evaluación y las tutorías se han realizado tanto presencialmente como RQdQH, a lo largo de 8 sesiones de 2 horas cada una. El grupo clase ha sido compuesto por 9 alumnos y alumnas.

La elección de la estrategia $\mathrm{ABPr}$ responde a la necesidad de ofrecer una metodología que prepare al alumnado para su inserción en el mundo laboral y, a su vez, favorezca un aprendizaje significativo de la materia específica de la asignatura impartida. Para ello, el ABPr resulta especialmente eficaz, ya que consiste en que los y las estudiantes elaboren un producto o artefacto propio de la disciplina, para dar respuesta a un reto (Du y Han, 2016); por ejemplo, en este caso deberán crear proyecto de enseñanzaaprendizaje que puedan aplicar, como docentes, en las aulas de Secundaria. Esta manera de proceder invita positivamente a la interdisciplinariedad y expone al alumnado a situaciones similares a las que encontrará en su futuro ámbito profesional (Rodríguez-Sandoval, Vargas-Solano, y Luna-Cortés, 2010). Otra de las virtudes de esta estrategia reside en su estructuración de trabajo -tanto dirigido, como autónomo- por equipos, mediante los cuales se deberá analizar el reto planteado e investigar y desarrollar el producto final de manera informada (Guisasola Aranzabal y Garmendia Mujika, 2014).

\section{Objetivo}

El objetivo principal de este trabajo es mostrar los beneficios educativos de la estrategia de enseñanzaaprendizaje $\mathrm{ABPr}$, en el ámbito de la formación superior de profesorado de música para Secundaria, en un contexto de docencia mixta o semipresencial. Para ello, se medirá la eficacia de dicha estrategia mediante la evaluación de los trabajos realizados por el alumnado y los resultados académicos que obtendrán los y las estudiantes al finalizar la asignatura.

\section{Desarrollo}

A continuación, se describirá detalladamente la innovación metodológica desarrollada, así como su aplicación a lo largo del primer semestre del curso académico 2020/21.

\subsection{Desarrollo de la innovación}

La innovación docente propuesta en este trabajo consiste en la aplicación de la estrategia de enseñanzaaprendizaje ABPr, en el ámbito de la educación superior. El desarrollo de dicha innovación consta de 3 etapas:

1. Planificación: Se plantea un reto a la clase y se especifican los productos o tareas que los y las estudiantes deberán desarrollar para resolverlo. Al tratarse de una estrategia basada en el trabajo en grupo, en esta fase también se forman los equipos de alumnos y alumnas y se establecerá un plan de trabajo.

2. Desarrollo: Durante esta fase, cada grupo de alumnos y alumnas elaborará los productos o tareas de manera autónoma e informada.

3. Exposición: En la última fase, cada grupo realiza la exposición de su proceso de trabajo y los productos o tareas realizadas. Para terminar, se procederá a la evaluación.

En la siguiente tabla se detallan acciones a realizar por docentes y estudiantes en cada una de las 3 etapas: 


$\begin{array}{lll}\text { Etapa Rol docente } & \text { Rol estudiante }\end{array}$

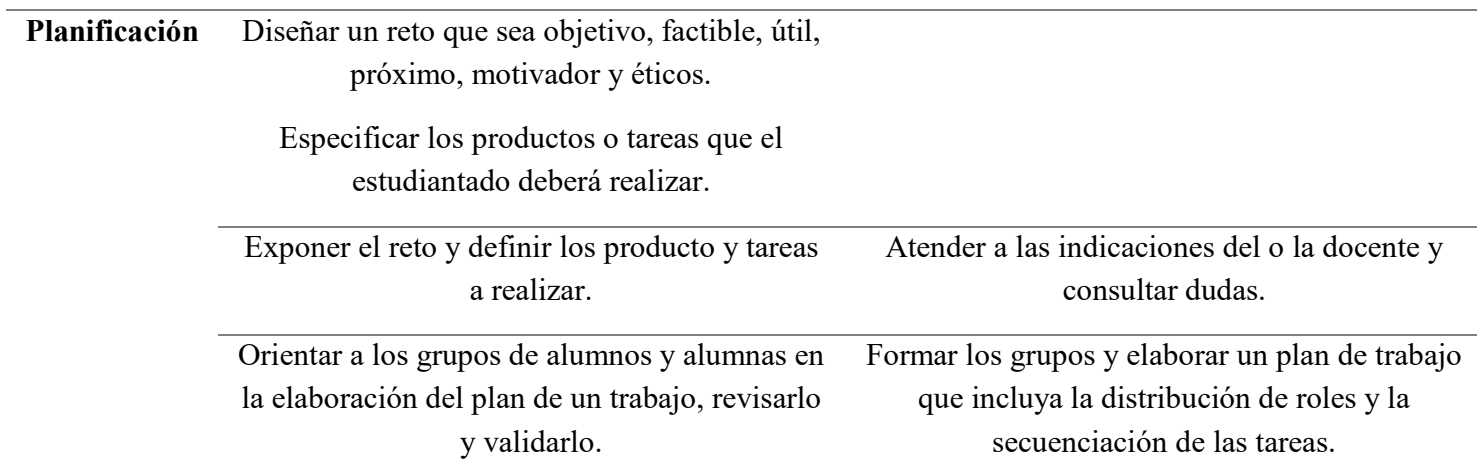

\begin{tabular}{|c|c|}
\hline Desarrollo & $\begin{array}{c}\text { Efectuar un seguimiento del trabajo de los y } \\
\text { las estudiantes, proponer ejemplo, atender sus } \\
\text { dudas y orientarles. }\end{array}$ \\
\hline
\end{tabular}

Investigar acerca de los producto o tareas encomendados.

Analizar los datos obtenidos y planificar la elaboración de los productos o tareas.

Elaborar los productos o tareas.

$\begin{array}{ccc}\text { Exposición } \quad \text { Atender a las exposiciones y comentarlas. } & \begin{array}{c}\text { Exponer, al profesorado y alumnado, los } \\ \text { productos o tareas realizados y su el }\end{array} \\ \text { procedimiento. }\end{array}$

Evaluar los proyectos y orientar a los alumnos y alumnas en los distintos recursos propuestos para su autoevaluación.
Efectuar una autoevaluación, coevaluación y/o heteroevaluación.

\subsection{Desarrollo de la asignatura}

La asignatura, planteada enteramente como proyecto, tiene como objetivo final la creación de una unidad didáctica, basada en la metodología ABPr, para la enseñanza de la expresión y la creación musicales en Secundaria. Para llevar a cabo esta tarea, los y las estudiantes deben aprender dicha metodología mediante la experimentación en primera persona, la investigación y el trabajo en grupo.

Las 2 primeras sesiones han servido para introducir la asignatura. En ellas, se ha procedido a explicar la teoría específica en torno a la didáctica de la expresión y la creación musicales, así como el funcionamiento que seguiría la asignatura. También se ha acompañado al alumnado en la formación de los grupos de trabajo -a cargo de los y las estudiantes-, se ha informado sobre el procedimiento de evaluación -rubricas de evaluación, autoevaluación, coevaluación y/o heteroevaluación, consensuadas entre alumnado y profesorado-, y se han puesto a disposición del alumnado herramientas de comunicación $R Q Q Q Q H$-entre estudiantes y con el profesorado- destinadas a gestionar los posibles conflictos en el grupo.

Las 6 sesiones posteriores se han dividido en dos mitades, de 60 minutos cada una, durante las que se han realizado, de manera práctica y en modalidad $R Q Q Q \mathrm{H}$ las siguientes actividades:

- La primera parte ha sido dedicada a implementar el proyecto de enseñanza-aprendizaje “Arqueología Musical”, previamente testado en la ESO. Aquí, los y las estudiantes de la universidad han participado, en primera persona y adoptando el rol de alumnos y alumnas de Secundaria, a un proyecto real que les ha servido para adquirir los recursos necesarios para llevar a cabo la elaboración de su propio proyecto. 
- En la segunda parte de cada sesión, los y las estudiantes se han dedicado a la creación del proyecto “Expresión y Creación Musical en la ESO". En este caso, han diseñado una unidad didáctica fundamentada en la metodología $\mathrm{ABPr}$, para la enseñanza de la expresión y la creación musicales en Secundaria.

Ambas actividades se han implementado con la técnica de ABPr y han constituido el $90 \%$ de la nota final de la asignatura. Este porcentaje se ha repartido entre 5 tareas entregadas a lo largo del curso, por valor de $10 \%$ de la asignatura cada una, y una exposición final, por valor del $40 \%$ restante, realizada virtualmente durante la octava y última sesión. También se ha evaluado, de manera continua, la participación e implicación en la asignatura, por un valor del $10 \%$ de la nota final.

\section{Resultados}

A continuación, se describen los proyectos "Arqueología Musical” y "Expresión y Creación Musical en la ESO", y se exponen su desarrollo y sus orientaciones de trabajo.

\subsection{Proyecto ${ }^{3} \$$ UXXHRTIDO XYFDO}

Para familiarizarse con la estrategia de enseñanza-aprendizaje $\mathrm{ABPr}$, los y las estudiantes han experimentado en primera persona una adaptación del proyecto "Arqueología Musical", originalmente diseñado para ser aplicado en la ESO, que consiste en inventar una cultura y crear los 5 objetos, o pistas, que permitirían a un arqueólogo conocer e interpretar su música:

- una partitura (con un sistema de notación de creación propia),

- una obra de arte plástica,

- un texto,

- un instrumento musical y

- una grabación (en vídeo o audio).

De este modo, quedan cubiertas diferentes facetas de la expresión y la creatividad musicales desde un punto de vista transversal y multidisciplinar. En el caso de su aplicación en la ESO, este proyecto culmina con una excursión al bosque, donde los grupos de alumnos y alumnas esconden sus objetos, en un primer tiempo, para luego realizar una segunda actividad donde deberán encontrar los objetos de alumnos y alumnas de otros grupos clase e intentar interpretar su significado. En el caso de su aplicación en la universidad, se prescinde de las actividades finales ya que se plantea únicamente cómo ejemplo para que los y las estudiantes puedan diseñar sus propios proyectos.

Este proyecto ha sido realizado por parejas. Al final de la sesión 2, los alumnos y alumnas han redactado un primer documento que incluía: nombre del grupo, componentes, planteamiento aproximado de la cultura inventada y sus objetos, plan de trabajo y cronograma. Este plan de trabajo debía tener en cuenta las fechas de entrega fijadas por el profesor o profesora. De modo autónomo y asincrónico, los y las estudiantes han debido definir los roles y responsabilidades que cada integrante debía ejercer, así como distribuir las tareas entre ellos y ellas.

La primera parte de las sesiones 3, 4, 5 y 6 se han dedicado a la creación de los objetos previamente mencionados y al aprendizaje de la teoría necesaria para llevarla a cabo. De este modo, se han trabajado conceptos de notación musical, composición, interpretación, organología, grabación, estética, musicología etc. Cada uno de estos objetos ha sido entregado y tenido en cuenta para la evaluación final de la asignatura, a través de tareas creadas específicamente en el Campus Virtual.

A continuación, se detallan las actividades realizadas a lo largo del proyecto: 
Actividad 0: invención una cultura. Imaginar una cultura inexistente, su hábitat, su época, su historia, sus rituales, su idioma, sus costumbres, sus roles, sus perspectivas de género, etc.

Actividad 1: creación de un instrumento. Se deberá fabricar un instrumento musical con material reciclado. Este instrumento deberá ser coherente con la cultura inventada y deberá poder ser interpretado con la partitura que se creará en una sesión posterior. Por lo tanto, habrá que hacer un trabajo de investigación sobre organología e interpretación instrumental. También será necesario investigar sobre las diferentes metodologías para enseñar nociones de organología en un contexto educativo, ya que el objetivo principal de esta actividad es el de poder potenciar la creatividad mediante la enseñanza de organología y de conjuntos instrumentales, así como de interpretación y expresión musical.

Actividad 2: creación de una partitura. Será necesario diseñar un sistema de notación, propio de la cultura inventada, con su simbología y sus normas. Por lo tanto, habrá que hacer un trabajo de investigación sobre los sistemas de notación existentes y su funcionamiento. También será necesario investigar sobre las diferentes metodologías para enseñar notación en un contexto educativo, ya que el objetivo principal de esta actividad es el de poder potenciar la creatividad mediante la enseñanza de la notación y el lenguaje musical.

Actividad 3: creación de un texto. Se deberá escribir un texto en el que quede descrita la cultura inventada, así como los usos y funciones de su música y sus rituales, haciendo referencia específicamente a la manera de tocar sus instrumentos y de leer sus partituras. También será necesario investigar sobre las diferentes metodologías para enseñar nociones musicológicas en un contexto educativo, ya que el objetivo principal de esta actividad es el de poder potenciar la creatividad mediante la enseñanza de las músicas de diferentes culturas.

Actividad 4: creación de una obra de arte plástica. Habrá que crear una obra de arte donde se represente algún aspecto de la música de la cultura inventada. Por ejemplo, podemos ver si la música se interpreta individualmente o en grupo, el contexto en el que se hace música, la manera en la cual se tocan los instrumentos, etc. En este caso, será necesario investigar sobre las diferentes metodologías para enseñar nociones de iconografía musical en un contexto educativo, ya que el objetivo principal de esta actividad es el de poder potenciar la creatividad mediante la enseñanza de las artes y su relación con las músicas de diferentes culturas.

Actividad 5: creación de una grabación. Se deberá gravar, en formato audio o vídeo, la interpretación de la canción escrita en la partitura, creada en la actividad 2, con el instrumento que se ha fabricado en actividad 1. Por lo tanto, habrá que hacer un trabajo de investigación sobre las técnicas y tecnologías para la grabación de música. También será necesario investigar sobre las diferentes metodologías para enseñar nociones de grabación musical en un contexto educativo, ya que el objetivo principal de esta actividad es el de poder potenciar la creatividad y expresividad musical mediante el uso de las TIC.

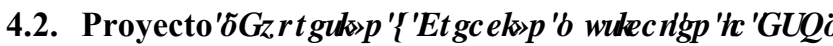

Este proyecto ha sido realizado por las mismas parejas que en el proyecto anterior. Al final de la sesión 2, los alumnos y alumnas también han redactado un primer documento que incluía: nombre del grupo, componentes, planteamiento del proyecto a aplicar en la ESO, curso al que irá destinado, plan de trabajo y cronograma. Al igual que con el proyecto anterior, este plan de trabajo debía tener en cuenta las fechas de entrega fijadas por el profesor o profesora.

Durante la segunda parte de las sesiones comentadas anteriormente, los grupos han puesto en práctica los conocimientos adquiridos con el proyecto "Arqueología Musical” y los han aplicado para la creación de su propio proyecto "Expresión y Creación Musical en la ESO". Por lo tanto, a medida que se han ido realizando las actividades del proyecto anterior, se ha procedido a diseñar las diferentes partes de una unidad 
didáctica, fundamentada en la metodología ABPr y destinada al alumnado de la ESO, que potencie la expresión y creación musical.

Finalmente, las unidades didácticas creadas han sido expuestas en la octava y última sesión del curso. Si la situación lo hubiese permitido, esta sesión hubiese sido presencial, pero finalmente, debido a las medidas adoptadas frente a la COVID19, se han realizado de manera virtual. Así, cada grupo de trabajo ha hecho una presentación de su unidad didáctica al resto de compañeros y profesores, a través de la herramienta BB Collaborate ${ }^{1}$. Las presentaciones en ningún caso han superado los diez minutos de duración y han contado con la intervención de todos los miembros de cada grupo de trabajo.

\section{Conclusiones}

Al organizar la asignatura de Didáctica de la Expresión y la Creación Musicales en torno a un proyecto, se ha logrado que los y las estudiantes adquieran un rol activo en la creación de conocimiento; se trata de aprender actuando, construyendo (Martínez et al., 2007). Además, al fundamentar el trabajo sobre retos reales y partir de la creación de recursos pertenecientes a su futuro laboral, se favorece el desarrollo de competencias específicas de la materia, más allá de la mera adquisición de conocimientos (Willard y Duffrin, 2003).

Por otro lado, la elevada calidad de los trabajos expuestos por los y las estudiantes, así como de los resultados obtenidos en las calificaciones finales de este curso -todas ellas situadas en la franja media alta del espectro de notas- y las observaciones del propio alumnado comunicadas al profesorado, prueban los efectos beneficiosos de la innovación en cuanto a formación, transmisión, motivación y satisfacción (Restrepo, 2005); reto difícil de alcanzar en una situación excepcional de semipresencialidad, como a la que se ha visto expuesto el actual sistema educativo.

Por ello, concluimos que la estrategia de enseñanza-aprendizaje ABPr, en el ámbito de la formación superior de profesorado de música para Secundaria, ha resultado ser una innovación educativa provechosa y muy bien adaptada al contexto de docencia semivirtual.

Finalmente, cabe hacer constar que la innovación ha sido presentada en un formato determinado y que se plantea como una herramienta metodológica flexible, abierta a adaptaciones para su adecuación a otras disciplinas, otros perfiles alumnado y otros contextos educativos.

\section{Referencias}

DU, X. y HAN, J. (2016) "A Literature Review on the Definition and Process of Project-Based Learning and Other Relative Studies”. \&UDUMH( GXFDMRQ núm. 7, pp. 1079-1083. Doi: 10.4236/ce.2016.77112

FINKEL, L. y CARPINTERO, E. (2019). "Diseño e implementación de un Plan de Formación del Profesorado de Educación Superior" en Perez Fuentes, M. C. (ed). , QQRIDFlyQ RFHQMHH, QYHWWDFIyQHQ( GXFDFIy QIMadrid: Editorial Dykinson. Pp. 785-798. Recuperado de: https://www.researchgate.net/profile/LucilaFinkel/publication/338718766_Diseno_e_implementacion_de_un_Plan_de_Formacion_del_Profesorado_de_Educaci on_Superior/links/5e272ee54585150ee775e8e7/Diseno-e-implementacion-de-un-Plan-de-Formacion-delProfesorado-de-Educacion-Superior.pdf

GUISASOLA ARANZABAL, G., y GARMENDIA MUJIKA, M. (2014). \$SLHQG]DNA EDWDR] HQ SLREGPDV]

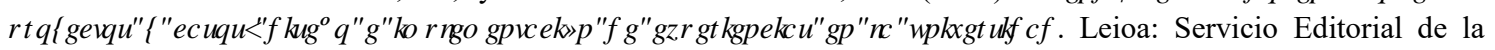
Universidad del País Vasco. Recuperado de: https://www.ehu.eus/documents/1870360/2202435/Eragin+Liburua+\%282\%29.pdf

\footnotetext{
${ }^{1}$ BB Collaborate es una herramienta de videoconferencias en tiempo real que permite crear ficheros, compartir aplicaciones y usar pizarras electrónicas virtuales para interactuar.
} 
LOSADA, S. G., y GARCÍA, M. Á. T. (2018). "Las estrategias didácticas en la práctica docente

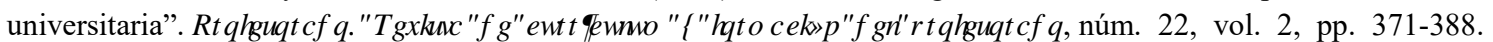
Doi: https://doi.org/10.30827/profesorado.v22i2.7728

MARTÍNEZ, F., HERRERO, L., GONZÁLEZ, J.M. y DOMÍNGUEZ, J.A. (2007). 3 LRNFFEDMGG(ODQQDIHSHUHQFH IQ IQXXWUDO HOFWRQFV DQG IQGXWUDO DSSCFDURQV GHJQ Universidad de Valladolid: Escuela Universitaria Politécnica. Recuperado de: http://157.88.123.53/articulos/EUP ProjectBased.pdf

RESTREPO, B. (2005). “Aprendizaje basado en problemas: una innovación didáctica para la enseñanza universitaria”. ( GXFDFy Q1 प्( GXFDCRUH, 8, 9-19. Recuperado de: https://dialnet.unirioja.es/servlet/articulo?codigo=2040741

RODRÍGUEZ-SANDOVAL, E., VARGAS-SOLANO, E. M. y LUNA-CORTÉS, J. (2010). "Evaluación de la estrategia aprendizaje basado en proyectos" HQ( GXFDFyQI (GXFDARUH, vol. 13, núm. 1, pp. 13-25. Recuperado de: https://www.redalyc.org/pdf/834/83416264002.pdf

WILLARD, K., y DUFFRIN, M.W. (2003). "Utilizing project-based learning and competition to develop student skills and interest in producing quality food ítems”. - RXLQDORII) RRG6FHQFH( GXFDMRQ núm. 2, pp. 69-73. 